\title{
Enhanced multiple-scattering and intra-half-cycle interferences in the photoelectron angular distributions of atoms ionized in midinfrared laser fields
}

\author{
Xiao-Min Tong,,${ }^{1,2, *}$ Predrag Ranitovic,,${ }^{3,4}$ Daniel D. Hickstein, ${ }^{3}$ Margaret M. Murnane, ${ }^{3}$ \\ Henry C. Kapteyn, ${ }^{3}$ and Nobuyuki Toshima ${ }^{1}$ \\ ${ }^{1}$ Division of Materials Science, Faculty of Pure and Applied Sciences, University of Tsukuba, 1-1-1 Tennodai, \\ Tsukuba, Ibaraki 305-8577, Japan \\ ${ }^{2}$ Center for Computational Sciences, University of Tsukuba,1-1-1 Tennodai, Tsukuba, Ibaraki 305-8573, Japan \\ ${ }^{3}$ JILA and Department of Physics, University of Colorado at Boulder, Boulder, Colorado 80309, USA \\ ${ }^{4}$ Lawrence Berkeley National Laboratory, 1 Cyclotron Road, Berkeley, California 94720, USA \\ (Received 28 January 2013; revised manuscript received 20 May 2013; published 15 July 2013)
}

\begin{abstract}
We investigate the physical mechanisms responsible for fine structure in the photoelectron angular distributions from atoms subject to intense midinfrared laser fields by solving the time-dependent Schrödinger equation in the integral form. By restricting the ionization to a half cycle of the laser field and then propagating the liberated electron wave packet during the laser pulse, we show conclusively that low-energy-momenta structure in the photoelectron angular distribution originates from multiple scatterings of the tunnel-ionized electron with the ion. We also show that two conditions must be satisfied simultaneously in order to observe prominent low-energy features. First, multiple scattering of the tunnel-ionized electron wave packet is necessary. Second, tunnel ionization must dominate over multiphoton ionization. While the first condition is generally satisfied for all laser wavelengths, the second condition is satisfied only for longer laser wavelengths.
\end{abstract}

DOI: 10.1103/PhysRevA.88.013410

PACS number(s): 32.80.Fb, 32.80.Qk, 34.80.Qb

An intriguing possibility driving research worldwide is the extent to which electrons extracted from a molecule by an intense femtosecond laser pulse carry information about the structure and dynamics of the molecule itself [1-3]. However, before this question can be fully addressed, a complete understanding of strong-field ionization (SFI) of atoms is needed. Both atoms and molecules illuminated with intense femtosecond laser fields exhibit a universal response, where they undergo strong-field ionization [4-8] and can also emit high-order harmonics of the fundamental laser field [9-11]. The recent ability to measure the full photoelectron angular distribution from atoms ionized by midinfrared (IR) laser fields provides a rich data set with which to compare experiment with theory, making it possible to uncover the physical mechanisms involved in SFI. Recent work explained some of the major features apparent in intense-field photoelectron spectra of atoms as resulting from electrons scattered by the Coulomb potential that can accumulate a different phase and interfere with electron trajectories that do not scatter $[12,13]$. However, other low-energy-momenta features that appear in the photoelectron angular distributions of atoms ionized by mid-IR lasers [14-26] were more challenging to explain.

Very recently, a simple and intuitive plane-spherical-wave (PSW) interference model [27] was used to explain most of the complex structures that appear in the photoelectron spectra of atoms ionized by mid-IR femtosecond lasers. The PSW model takes into account the quantum phase accumulated by the electron wave packet, which depends on the particular trajectory or journey it takes in the laser field. In this picture, a returning electron wave packet, driven by the laser field, passes by the parent ion (plane wave) and interferes with the part of the wave packet that scatters off the parent ion (spherical wave).

\footnotetext{
*tong.xiaomin.ga@u.tsukuba.ac.jp
}

Thus, the shape and spacing of the photoelectron interference structures directly correspond to the specific number of times the electron reencounters its parent ion before scattering strongly. However, while the PSW model explained most of the major features in the photoelectron momentum distributions, such as the low-energy parabolic nodal (spiderlike) structures centered around the polarization axis, additional rich structural features [moderate longitudinal oscillations; see Fig. 1(a) in Ref. [27] or Fig. 1(d) in Ref. [20]] at higher energy-momenta were not explained.

In this work, by solving the time-dependent Schrödinger equation in the integral form, we show quantitatively that the low-energy features in mid-IR strong-field ionization photoelectron angular distributions are due to multiple rescattering of the electron in the laser field. We also explain why these interferences are only observed experimentally for long driving laser wavelengths. Moreover, we find that the features appearing as moderate oscillations in the photoelectron distribution at higher energy are due to intra-half-cycle interferences (see Fig. 1).

The photoelectron angular distributions created during strong-field ionization can be thought of as originating from two sequential interference processes: (1) interferences of electron wave packets created in a particular half-cycle field and further propagated in the remaining laser field and (2) interferences between the electron wave packets created in different half cycles. The first process provides the most dynamical information and gives rise to the global structure, as shown in Fig. 1(a). The second process gives rise to the detailed structure in the photoelectron angular distribution, such as the peak positions and widths, as shown in Fig. 1(b).

To identify the mechanisms responsible for moderate longitudinal oscillations (ellipses in Fig. 1), and to show from first principles that fine structure at low momenta is due to multiple scattering of the electron, we explore photoelectron 


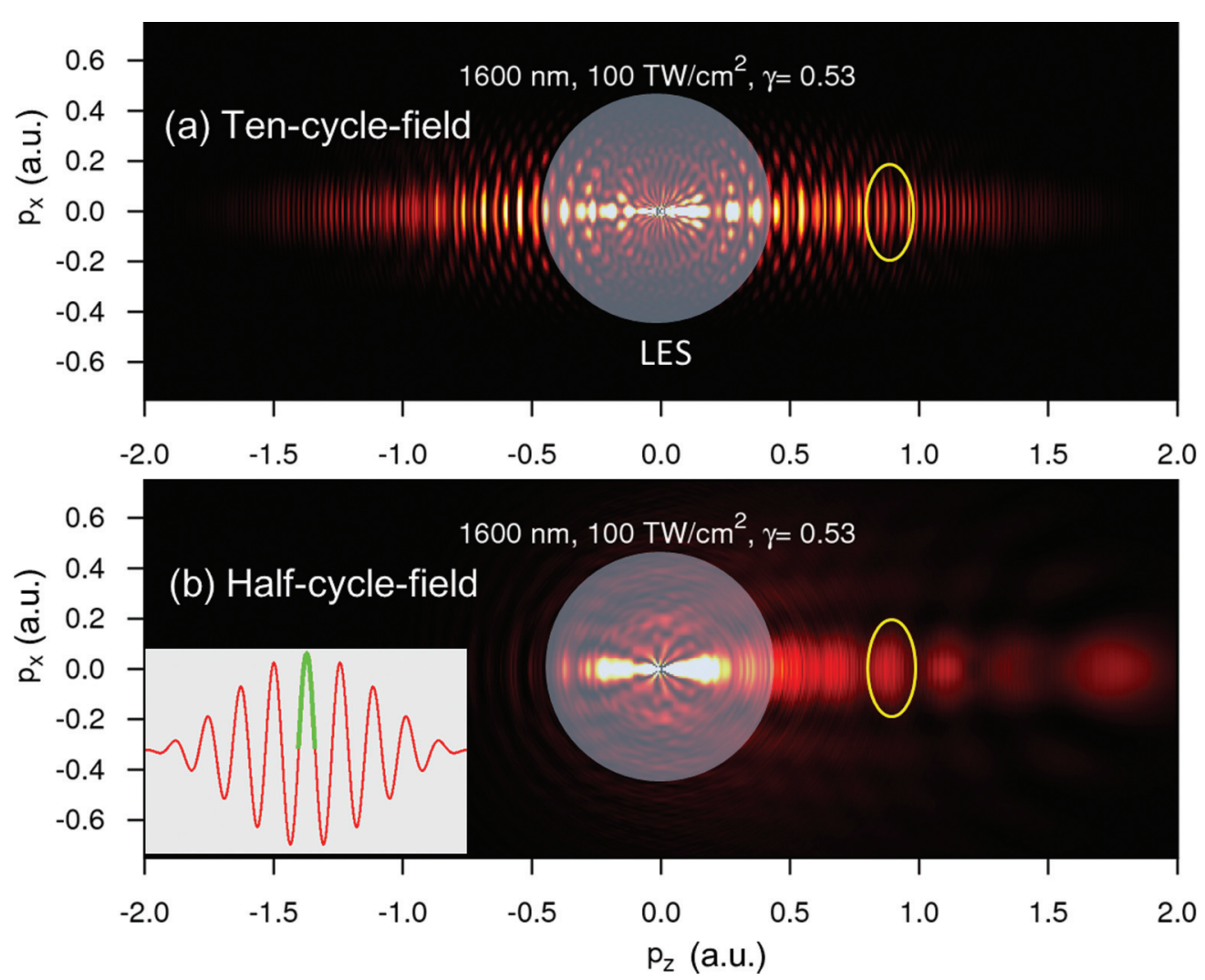

FIG. 1. (Color online) Photoelectron momentum spectra of $\mathrm{H}$ atoms in a mid-IR laser field for the case of (a) a half-cycle pulse at the peak of the field and (b) a ten-cycle pulse. Inset: The full pulse, with the peak half cycle marked in green. The low-energy structures are highlighted by transparent circles, while the moderate oscillations are marked by ellipses.

distributions from a given half cycle of the driving laser field. To do this, we solve the time-dependent Schrödinger equation in the integral form [28,29], which allows us to separate the "creation" of the electron wave packet from its propagation (atomic units $\hbar=e=m_{e}=1$ are used):

$$
\begin{aligned}
\Psi(\infty) & =-i \int_{-\infty}^{\infty} e^{-i \int_{t}^{\infty} H\left(t^{\prime}\right) d t^{\prime}} V_{\mathrm{ext}}(t) e^{-i H_{0} t} \Psi_{0} d t \\
& =\sum_{k=-\infty}^{\infty} \Psi_{k},
\end{aligned}
$$

with

$$
\Psi_{k}=-i \int_{t_{k}}^{t_{k+1}} e^{-i \int_{t}^{\infty} H\left(t^{\prime}\right) d t^{\prime}} V_{\mathrm{ext}}(t) e^{-i H_{0} t} \Psi_{0} d t .
$$

Equation (1) shows the fact that the final total electron wave function is a coherent superposition of the electron wave packet "created" between the $t_{k}$ and $t_{k+1}$ time period and then propagated in the combined atomic and remaining laser fields; $H(t)=H_{0}+V_{\text {ext }}(t)$ is an atomic Hamiltonian in an external laser field; $H_{0}$ is the laser-field-free atomic Hamiltonian; $V_{\text {ext }}(t)$ is the atomic-laser interaction in the velocity gauge,

$$
V_{\mathrm{ext}}(t)=-\boldsymbol{p} \cdot \boldsymbol{A}(t)+A^{2}(t) / 2
$$

and the vector potential $\boldsymbol{A}(t)$ is expressed as

$$
\boldsymbol{A}(t)=\int_{t^{\prime}}^{\infty} \boldsymbol{E}\left(t^{\prime}\right) d t^{\prime}
$$

For this study, we use the velocity gauge because of convergence considerations [7,30]. The laser field is expressed as

$$
\boldsymbol{E}(t)= \begin{cases}E_{0} \hat{\boldsymbol{\epsilon}} \cos (\omega t) \cos ^{2}\left(\frac{t}{\tau} \pi\right) & -\frac{\tau}{2} \leqslant t \leqslant \frac{\tau}{2} \\ 0 & \text { otherwise }\end{cases}
$$

where $E_{0}$ is the laser peak field strength, $\hat{\boldsymbol{\epsilon}}$ is the unit vector of laser polarization direction, $\omega$ is the laser center frequency, and $\tau$ is the pulse duration.

Because a laser field is periodic, the dynamics that occurs within each half cycle is similar apart from a phase due to the time shift. The final electron momentum distribution is a coherent sum of each half-cycle contribution. Thus, to understand physical origins of the rich features in the photoelectron distributions in mid-IR laser fields, we focus on the ionization process during the half-cycle period at the peak of the laser pulse, for a laser wavelength of $1600 \mathrm{~nm}$ and intensity $10^{14} \mathrm{~W} / \mathrm{cm}^{2}$, with a pulse duration of ten optical cycles, as shown in Fig. 2. Just after the ionization during the peak half cycle of the laser field (Fig. 2, IQ1 and IQ2), according to the definition of tunnel ionization, any electron wave packet located in the outer region $\left(r \geqslant r_{c}\right.$, with $r_{c}$ the classical tunneling position from the center) comes from tunnel ionization. In contrast, an electron wave packet located in the inner region $\left(r<r_{c}\right)$ does not contribute to tunnel ionization and can be ionized by the remaining laser field and contaminate the rescattering wave packet. This part can be assigned to 


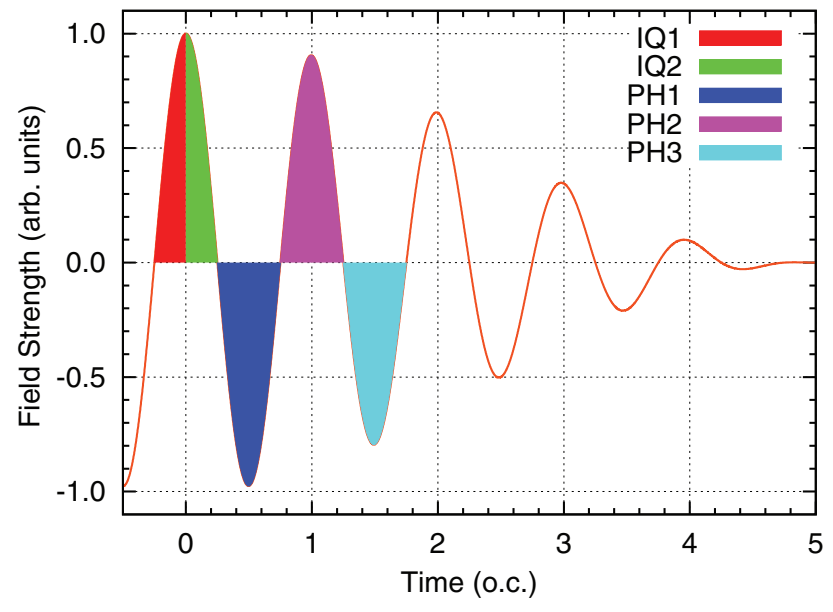

FIG. 2. (Color online) Midinfrared laser field strength. Ionization happens in the half-cycle period, which is further recast into the first quarter cycle (IQ1) and second quarter cycle (IQ2) ionization. PH1, $\mathrm{PH} 2, \mathrm{PH} 3, \ldots, n$ represent the ionized electrons propagated in the laser field up to $1,2,3, \ldots, n$ half cycles.

multiphoton absorption (including excitation and ionization). To eliminate the contribution from the multiphoton absorption processes during the peak half cycle, we remove the electron wave packet from the inner region close to the core after IQ2. Note that a similar method was successfully used to obtain all the details of the rescattering process in a full nonperturbative quantum simulation [29]. This may justify the validity of the present method used to separate tunnel ionization from multiphoton ionization approximately.

Figure 1 shows photoelectron momentum spectra created only during the peak half cycle of the laser field and propagated in the remaining pulse [Fig. 1(a)] and from the whole ten-cycle pulse [Fig. 1(b)]. We see the expected strong yield along the laser polarization direction due to tunnel ionization for both cases. However, it is interesting and informative to compare the global patterns of the low- (transparent circles) and highenergy (ellipses) structures highlighted. Due to interferences between electron wave packets ionized from different half cycles, the ten-cycle spectra have more complex and detailed low-energy spiderlike structures than the half-cycle spectra. At intermediate energy-momenta $\left(P_{z}>0.5\right.$ a.u.), the electron density is mainly distributed along the polarization direction, with moderate density modulations appearing in the half-cycle case. These oscillations still exist in the ten-cycle field but are more difficult to see because of the presence of many sharp peaks due to interferences from different half cycles. Therefore, we focus now on the physical mechanisms giving rise to the features observed in the case of half-cycle ionization and subsequent propagation.

To investigate the contribution of multiple rescattering, we turn on (or off) the laser field at a certain time period after ionization, in half-cycle intervals. To investigate the origin of the moderate oscillations, we further analyze ionization from each quarter cycle of the half-cycle field. Figure 3 plots results from the ionization process restricted only to the peak half cycle of the laser field and the electron wave-packet propagation in the remaining laser field (PHr). To illustrate the important physical processes, we also show the photoelectron spectra when the laser field is turned off immediately after the ionization (PH0), after one half cycle (PH1), after four half cycles (PH4), and after seven half cycles (PH7). We see that the low-energy peaks disappear if we turn the laser field off immediately after ionization, whereas oscillations at intermediate and high energies still appear. This demonstrates conclusively that the low-energy peaks are associated with electron wave-packet propagation or rescattering processes in multiple half cycles of the laser field, consistent with the PSW interference model. By including the next half cycle of the laser field (Figs. 2 and 3, PH1), the amplitude of the high-energy oscillation is enhanced, while peaks still do not emerge at the lower energies. As we include an increasing number of subsequent half cycles (Fig. 3, PH4 and PH7), lower-energy peaks eventually appear. These calculations therefore confirm that the low-energy fine structure originates from the multiple rescattering of the electron wave packet ionized and driven in a long wavelength mid-IR laser field.
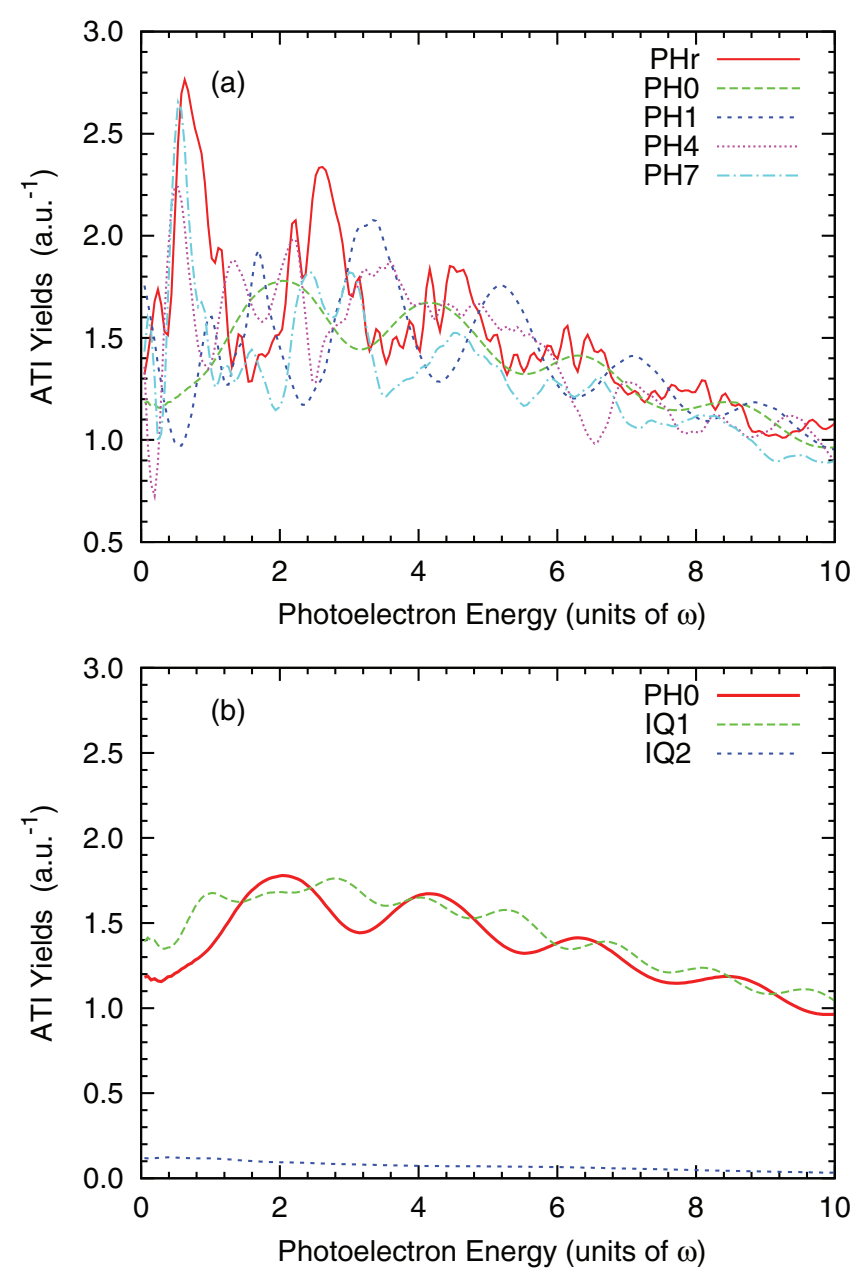

FIG. 3. (Color online) (a) Photoelectron spectra of $\mathrm{H}$ atoms ionized in a half-cycle field and propagated in different later fields. PH0 means the laser field is turned off immediately after the half-cycle ionization and $\mathrm{PHr}$ means the electron propagated in all the remaining laser field. (b) Photoelectron spectra of $\mathrm{H}$ atoms ionized during the first and second quarter cycles without further propagation in the laser field. 
After turning the laser field off immediately after the ionization occurs at the peak of the laser field, we expect that the photoelectron spectra will become smooth since the rescattering process is turned off. Figure 3(a) ( $\mathrm{PHO}$ ), however, shows moderate oscillations in the energy domain. To investigate the origin of the higher-energy oscillations, we further recast ionization into the first and second quarters (IQ1, IQ2) of the peak half cycle as depicted in Fig. 2. Note that we classify the electron ionization in the IQ1 (IQ2) by the integration period in Eq. (2). The electron may actual be ionized at a later time. Our results are shown in Fig. 3(b). Here we see that ionization mainly comes from the first quarter cycle and that the yield oscillations are smeared. We also see that the ionization yield from the second quarter cycle is about $10 \%$ and that the oscillations are gone. Thus we conclude that the interference between the EWPs ionized during the first and second quarter cycles is responsible for the oscillations of the total yield in the energy domain as seen in Fig. 3(b) (PH0). The larger contribution of IQ1 can be understood from the fact that the IQ1 field not only ionizes the atom but also significantly excites the ground-state population, which can be further ionized by the IQ2 field [see Eq. (2)]. This results in a large yield from the IQ1 period and gives rise to the oscillations of the electron yield. For IQ2, such a process is suppressed.

This interference results in the moderate longitudinal oscillation marked by the ellipses in Fig. 1. Since this interference results from electrons that tunnel during the same half cycle, we call it "intra-half-cycle interference." These intra-half-cycle interferences are different from the intracycle interferences that result from electrons that tunnel at the same vector potential and create oscillations [31] and carpetlike structures [32].

In principle, the same low- and high-energy interference structures should also appear in photoelectron spectra generated by shorter laser wavelengths. Thus, in Fig. 4, we compare the photoelectron spectra generated in a half-cycle period and propagated in the remaining laser fields ( $\mathrm{PHr}$ ) for a given laser intensity $\left(10^{14} \mathrm{~W} / \mathrm{cm}^{2}\right)$ [Fig. 4(a)] or a given Keldysh parameter $\left[\gamma=\sqrt{I_{P} /\left(2 U_{p}\right)}=0.75\right.$, with $I_{p}$ the ionization potential of the atom and $U_{p}$ the pondermotive energy of the electron in the laser field] [Fig. 4(b)] with different laser wavelengths and a pulse duration of ten optical cycles. We also plot the photoelectron spectra if the laser field is turned off immediately after ionization (PH0). The difference between the two can be attributed to multiple scatterings. For the laser intensity shown in Fig. 4(a), we see that (1) the oscillation amplitude due to intra-half-cycle interference (PH0) decreases as the laser wavelength decreases; (2) the relative magnitude of the lower-energy peaks due to multiple scattering also decreases as the laser wavelength decreases; and (3) the strength of the photoelectron spectra decreases as the laser wavelength decreases (the tunneling time is shorter for shorter wavelengths). For the Keldysh parameter shown in Fig. 4(b), both the general pattern and the yields are almost independent of laser wavelength. This also explains that in SFI the lower-energy structures $[27,33]$ mainly depend on the Keldysh parameter. From this observation, we conclude that low-energy photoelectron peaks should also be observed for 800-nm and even 400-nm laser fields, not necessarily only for mid-IR laser fields. However, experiments to date have
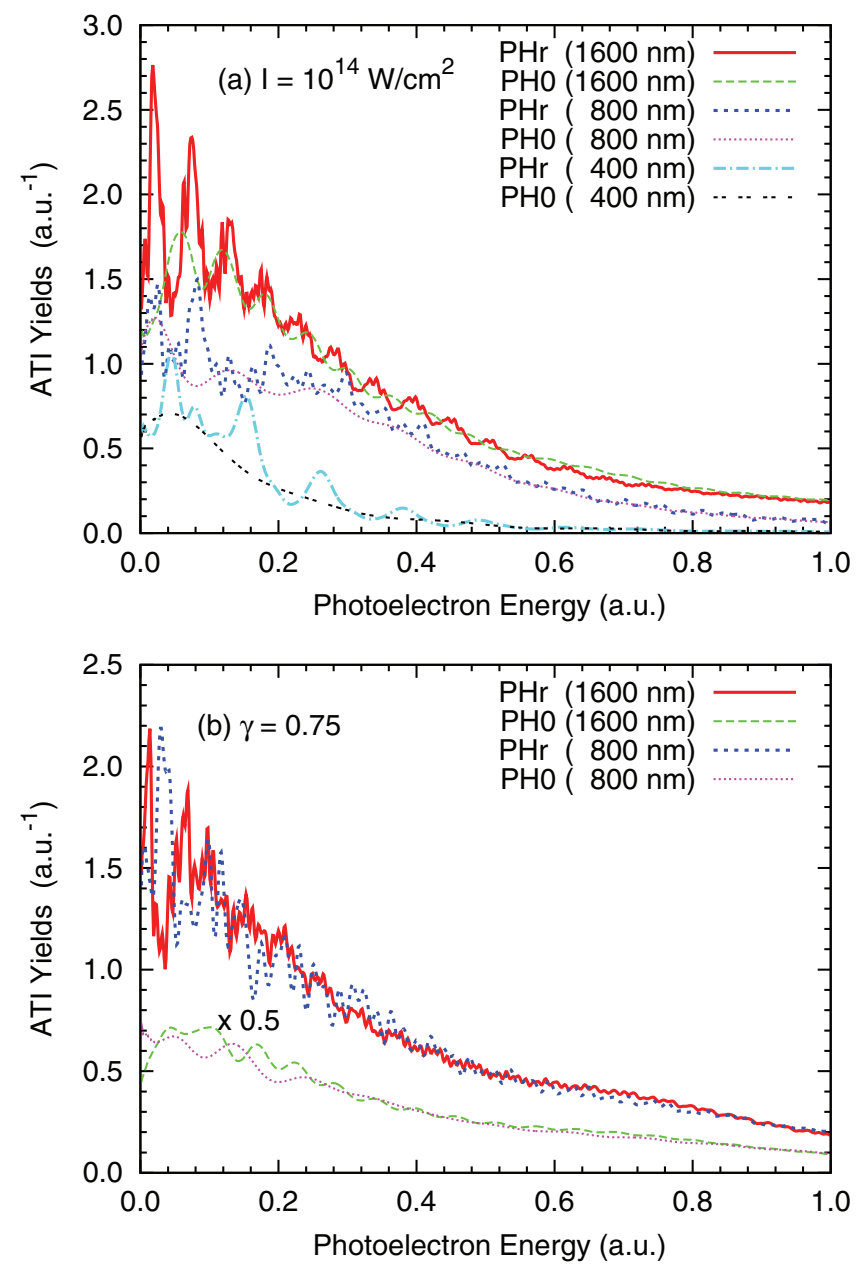

FIG. 4. (Color online) Photoelectron spectra of $\mathrm{H}$ atoms ionized in a half cycle and propagated in the remaining laser field (PHr) or no laser field after the ionization (PH0) for (a) the same laser intensity and (b) the same Keldysh parameter; $\gamma=0.75$ with different laser wavelengths.

not reported such low-energy features for wavelengths below $800 \mathrm{~nm}$.

We emphasize that the above analysis makes it possible to separate the contributions of tunnel ionization that happens in the half-cycle laser field, from multiphoton ionization. To understand the discrepancies between experiment and theory, we separate tunnel ionization from multiphoton ionization. We note that after a half-cycle ionization period there are still low-energy electron wave packets that result from multiphoton ionization. Thus, the final photoelectron spectra after half-cycle ionization can be separated into two parts: electron wave packets due to multiphoton ionization at short distances

TABLE I. The ratio of the tunnel ionization probability to the total ionization probability ionized in a half cycle $\left(I_{0}=10^{14} \mathrm{~W} / \mathrm{cm}^{2}\right)$.

\begin{tabular}{lllll}
\hline \hline & $0.5 I_{0}$ & $1.0 I_{0}$ & $1.5 I_{0}$ & $2.0 I_{0}$ \\
\hline $400 \mathrm{~nm}$ & 0.055 & 0.391 & 0.614 & 0.727 \\
$800 \mathrm{~nm}$ & 0.570 & 0.812 & 0.931 & 0.969 \\
$1600 \mathrm{~nm}$ & 0.970 & 0.995 & 1.000 & 1.000 \\
\hline \hline
\end{tabular}


and electron wave packets due to tunnel ionization at distances greater than $r_{c}$. In Fig. 4, we exclude contributions due to multiphoton ionization. Table I shows the ratio of the tunnel ionization probability to the total ionization probability for half-cycle ionization, which explains why the prominent low-energy features were experimentally observed only in the long-wavelength regime. Note that in Fig. 4(b), for the given Keldysh parameter, the laser intensity of $800 \mathrm{~nm}$ is $2 \times$ $10^{14} \mathrm{~W} / \mathrm{cm}^{2}$. For such an intensity, ionization is dominated by over-the-barrier ionization [34], not tunnel ionization. We see that two conditions have to be satisfied simultaneously in order to observe prominent low-energy features: first, multiple scattering of the tunnel-ionized electron wave packet is necessary; second, tunnel ionization must dominate over multiphoton ionization. While the first condition is satisfied more or less for all laser wavelengths, the second condition is satisfied only for longer laser wavelengths (see Table I). This is also illustrated in Fig. 4(a), where we see that the relative modulation and photoelectron yields for shorter wavelengths (i.e., 400 and $800 \mathrm{~nm}$ ) are weaker than for a longer wavelength $(1600 \mathrm{~nm})$, while the total ionization rates for short wavelengths are much larger than for long wavelengths. When the contributions of multiphoton ionization and tunnel ionization are comparable, the final photoionization spectra can come from multiphoton ionization, tunnel ionization, and the interference between the two. This is the reason why we can only separate the two processes at the wave-function level, not the photoionization spectra level.

In conclusion, by solving the time-dependent Schrödinger equation in the integral form, and analyzing the ionization in a half-cycle of the laser field, we showed conclusively that low-energy-momenta structure in the photoelectron angular distribution originates from multiple scatterings. We also explained that the lower-energy structures are only observed experimentally for long driving wavelengths because multiphoton ionization also plays an important role for shorter laser wavelengths. Furthermore, we attribute the moderate longitudinal oscillations at higher electron energy-momenta to intra-half-cycle interference of the electron wave packets.

This research was supported by a Grand-in-Aid for Scientific Research from the Japan Society for the Promotion of Science and the HA-PACS project for advanced interdisciplinary computational sciences by exa-scale computing technology. D.H., M.M., and H.K. acknowledge support from the DOE Office of Basic Energy Sciences (AMOS program).
[1] J. Itatani, J. Levesque, D. Zeidler, H. Niikura, H. Pepin, J. C. Kieffer, P. B. Corkum, and D. M. Villeneuve, Nature (London) 432, 867 (2004).

[2] W. Li, A. A. Jaro-Becker, C. W. Hogle, V. Sharma, X. Zhou, A. Becker, H. C. Kapteyn, and M. M. Murnane, Proc. Natl. Acad. Sci. USA 107, 20219 (2010).

[3] C. Blaga, J. Xu, A. DiChiara, E. Sistrunk, K. Zhang, P. Agostini, T. Miller, L. DiMauro, and C. Lin, Nature (London) 483, 194 (2012).

[4] L. V. Keldysh, Sov. JETP 47, 1945 (1964).

[5] F. H. M. Faisal, J. Phys. B 6, L89 (1973).

[6] M. Lewenstein, P. Balcou, M. Y. Ivanov, A. LHuillier, and P. B. Corkum, Phys. Rev. A 49, 2117 (1994).

[7] D. Bauer, D. B. Milosevic, and W. Becker, Phys. Rev. A 72, 023415 (2005).

[8] D. B. Milosevic, G. G. Paulus, D. Bauer, and W. Becker, J. Phys. B 39, R203 (2006).

[9] J. L. Krause, K. J. Schafer, and K. C. Kulander, Phys. Rev. Lett. 68, 3535 (1992).

[10] P. B. Corkum, Phys. Rev. Lett. 71, 1994 (1993).

[11] Z. Chang, A. Rundquist, H. Wang, M. M. Murnane, and H. C. Kapteyn, Phys. Rev. Lett. 79, 2967 (1997).

[12] Y. Huismans et al., Science 331, 61 (2011).

[13] X.-B. Bian, Y. Huismans, O. Smirnova, K.-J. Yuan, M. J. J. Vrakking, and A. D. Bandrauk, Phys. Rev. A 84, 043420 (2011).

[14] C. I. Blaga, F. Catoire, P. Colosimo, G. G. Paulus, H. G. Muller, P. Agostini, and L. F. DiMauro, Nat. Phys. 5, 335 (2009).

[15] F. H. M. Faisal, Nat. Phys. 5, 319 (2009).

[16] W. Quan et al., Phys. Rev. Lett. 103, 093001 (2009).

[17] C. Y. Wu, Y. D. Yang, Y. Q. Liu, Q. H. Gong, M. Wu, X. Liu, X. L. Hao, W. D. Li, X. T. He, and J. Chen, Phys. Rev. Lett. 109, 043001 (2012).
[18] I. Burenkov, A. Popov, O. Tikhonova, and E. Volkova, Laser Physics Letters 7, 409 (2010).

[19] C. Liu and K. Z. Hatsagortsyan, Phys. Rev. Lett. 105, 113003 (2010).

[20] T.-M. Yan, S. V. Popruzhenko, M. J. J. Vrakking, and D. Bauer, Phys. Rev. Lett. 105, 253002 (2010).

[21] M.-H. Xu, L.-Y. Peng, Z. Zhang, Q. Gong, X.-M. Tong, E. A. Pronin, and A. F. Starace, Phys. Rev. Lett. 107, 183001 (2011).

[22] D. A. Telnov and S.-I. Chu, Phys. Rev. A 83, 063406 (2011).

[23] A. Kästner, U. Saalmann, and J. M. Rost, Phys. Rev. Lett. 108, 033201 (2012).

[24] P. A. Korneev, S. V. Popruzhenko, S. P. Goreslavski, W. Becker, G. G. Paulus, B. Fetic, and D. B. Milosevic, New J. Phys. 14, 055019 (2012).

[25] C. Lemell, K. I. Dimitriou, X.-M. Tong, S. Nagele, D. V. Kartashov, J. Burgdörfer, and S. Gräfe, Phys. Rev. A 85, 011403 (2012).

[26] C. Lemell, J. Burgdörfer, S. Gräfe, K. I. Dimitriou, D. G. Arbó, and X.-M. Tong, Phys. Rev. A 87, 013421 (2013).

[27] D. D. Hickstein et al., Phys. Rev. Lett. 109, 073004 (2012).

[28] X. M. Tong, K. Hino, and N. Toshima, Phys. Rev. A 74, 031405 (2006).

[29] X. M. Tong, S. Watahiki, K. Hino, and N. Toshima, Phys. Rev. Lett. 99, 093001 (2007).

[30] E. Cormier and P. Lambropoulos, J. Phys. B 29, 1667 (1996).

[31] D. G. Arbó, E. Persson, and J. Burgdörfer, Phys. Rev. A 74, 063407 (2006).

[32] P. A. Korneev et al., Phys. Rev. Lett. 108, 223601 (2012).

[33] L. Guo et al., Phys. Rev. Lett. 110, 013001 (2013).

[34] X. M. Tong and C. D. Lin, J. Phys. B 38, 2593 (2005). 